Reprod. Nutr. Dévelop., 1984, 24 (5 B), 671-692.

\title{
Les mécanismes de régulation du bilan d'énergie
}

\section{J. LE MAGNEN}

Laboratoire de Neurophysiologie sensorielle et comportementale, Collège de France, C.N.R.S.,

11, place Marcelin Berthelot, 75231 Paris Cedex 05.

\section{Summary. The mechanisms of regulation of body energy balance.}

To establish energy balance, the three distinct terms of the balance must be precisely defined and measured. These terms are : the energy input by food intake, energy expenditure as heat and the body energy content in the form of lean and fat masses. The constancy of this fat store, generally associated with that of body weight, is an indication of the balance between energy intake and the sum of expenditure and non-lipidic energy retention. This balance is only carried out at medium or long-term. It results from the initiation of mechanisms regulating strongly positive or negative short-term balances between gains and losses. Four possible models of this regulation are presented and discussed using experimental data. A number of works is reported, demonstrating the role played by two complementary mechanisms of regulation. The first one is neuroendocrine mechanism controlling food intake and adjusting it to the input and output of glucose in the blood compartment. The second one is an autonomous mechanism regulating the body fat mass. It corrects, at medium and long-term, the filling and depletion of the adipose compartment which result from imbalances in the overall energy balance. This mechanism indirectly affects food intake in a regulatory sense. It is neurally controlled by the sympathetic and parasympathetic nervous system and governed by the medioventral nuclei of the hypothalamus.

Depuis Claude Bernard, "Physiologie » est synonyme d'« Etude des mécanismes de régulation ". A ce titre, l'étude de la régulation du bilan d'énergie peut être considérée comme un sommet de la physiologie. Tous les systèmes cellulaires et organes et leurs ensembles fonctionnels sont soumis à des mécanismes régulateurs particuliers. Ils sont tous incorporés à et dépendants d'un mécanisme régulateur intéressant l'organisme en son entier, mécanisme qui contrôle son acquisition et ses pertes d'énergie, et en assure l'équilibre.

\section{Le bilan d'énergie.}

Rappelons brièvement quels sont les trois termes du bilan d'énergie. L'entrée d'énergie E est constituée par la prise d'aliments. Elle est, on le sait, essentielle- 
ment périodique. Elle se mesure soit par le volume des épisodes alimentaires successifs ou " repas » et par l'intervalle de temps qui les sépare, qui constituent la " séquence alimentaire ", soit par la prise cumulée sur un temps donné. II s'agit alors de la mesure du " débit d'entrée " d'énergie. Elle s'exprime en termes d'énergie métabolisable apportée par les 3 macronutriments que l'on mesure en bombe calorimétrique. La dépense énergétique, ou thermogenèse $T$, se mesure, on le sait, par calorimétrie directe ou indirecte. Les composantes de ce débit d'énergie sont bien connues : le métabolisme dit « de base » de l'animal à jeun, au repos et dans la zone de neutralité thermique, aúquel s'ajoutent (pour faire varier considérablement ce débit) l'incrément de chaleur alimentaire, l'exercice et, en ambiance froide, la thermogenèse de réchauffement. Le troisième terme est le contenu corporel en énergie (CCE). Ce dernier comporte deux composantes à distinguer dans l'établissement du bilan. La première est la masse dite " maigre " qu'il serait préférable de désigner " masse de structure ». Elle est constituée par la masse protéique (essentiellement musculaire) les lipides de structure et les molécules de haute énergie, sources, entre autres, des synthèses enzymatiques et hormonales. Le gain de cette masse et la rétention d'énergie qu'elle implique sont " fonctionnels » dans la croissance et la gestation, dans le développement musculaire par l'exercice. Les pertes de ce compartiment sont représentées par les excrétions : pertes fécales et urinaires, allaitement, par exemple. Ces gains et pertes de cette composante dite maigre du CCE sont désignés plus loin $\triangle \mathrm{M}$. La seconde composante est la masse adipeuse. II est bien connu qu'avec cette réserve lipidique représentant en moyenne chez l'homme 15 à $20 \%$ du poids corporel, nous disposons en nous de trois mois de vivres. Cette réserve interne d'énergie métabolisable, comme toute réserve, est alternativement utilisée et reconstituée. Le bilan particulier des entrées et sorties de ce compartiment $(\Delta \mathrm{G})$ est un élément majeur à prendre en considération dans l'étude et la mesure du bilan global.

On en conclut aussi que cet équilibre global a été associé à celui d'un bilan particulier des entrées et sorties du compartiment de réserve.

On dit alors, communément et assez grossièrement, que l'équilibre du bilan a été associé ou a conduit à une régulation du poids corporel. Notons en passant que cette notion de régulation du poids, substituée à celle de régulation du bilan énergétique (notion chère aux spécialistes de l'obésité) est sommaire. Le poids n'est pas, bien évidemment, régulé en soi. Personne n'a jamais montré qu'après un séjour prolongé en apesanteur, un astronaute avait grossi de $500 \mathrm{~kg}$ pour com-

(1) $\mathrm{Et}=\mathrm{Tt}+(\Delta \mathrm{M}+\Delta \mathrm{G})$

(2) $E^{*} \mathrm{t}-\left(\Delta \mathrm{M}^{*}+\Delta \mathrm{G}\right)^{*}=T \mathrm{t}$

(3) $\mathrm{Et}-\mathrm{Tt}=\Delta \mathrm{M}^{*}+\Delta \mathrm{G}^{*}$

(4) si $\Delta \mathrm{G}=0 \mathrm{Et}=\mathrm{Tt}-\Delta \mathrm{M}$

$E=$ Débit d'entrée d'énergie - Prise alimentaire ; $T=$ Débit de sortie d'énergie - Thermogenèse ; $\mathrm{t}=$ temps de mesure; $\Delta \mathrm{M}=$ Gain ou perte d'énergie de la masse maigre; $\Delta \mathrm{G}=\mathrm{Gain}$ ou perte d'énergie de la masse lipidique ${ }^{*}=$ paramètre mesuré. 
penser sa perte totale de poids. D'autre part, la masse des graisses, bien qu'elle soit la part la plus fluctuante en tant que réserve du poids total du corps, n'en est pas, bien entendu, la seule composante. La constante de la charge en eau $170 \%$ du poids) est assurée par des mécanismes particuliers de régulation indépendants de ceux du bilan d'énergie. Les graisses, on l'a vu, ne sont pas non plus les seuls composants du contenu corporel en énergie. La charge en eau étant constante, l'adiposité peut s'élever parallèlement à une réduction du tissu maigre et le poids demeurer constant. La mesure d'un gain ou d'une perte de poids ou sa constance ne peuvent donc être qu'une indication de l'équilibre du bilan, toutes choses étant égales par ailleurs.

Cependant, l'observation de la constance du poids sur une longue période vérifie l'équilibre réalisé à moyen ou long terme entre thermogenèse et ingestion. Elle donne aussi une indication de la précision des mécanismes régulateurs assurant, dans le délai considéré, l'ajustement des entrées et sorties d'énergie. On a souvent cité le calcul suivant lequel un sujet humain aura grossi en 30 ans de $20 \mathrm{~kg}$ s'il prend chaque jour durant ces 30 ans un excès quotidien de calories sur ses dépenses portant seulement sur un morceau de sucre.

\section{Relations entre entrées et dépenses d'énergie.}

Cet équilibre du bilan déduit de la mesure des trois termes ou de l'un d'entre eux, tel que le poids, n'est jamais constaté qu'à moyen ou long terme. A court terme, le gain d'énergie par l'ingestion, comme la thermogenèse, varie considérablement. Dans la mesure où ses variations ne sont pas synchrones, le troisième terme (c'est-à-dire la masse de réserve de graisse) jouant son rôle de "ballast " $d^{\prime}$ énergie varie également en gain ou perte. A court terme, des situations extrêmes de bilans positifs ou négatifs, d'excès ou de déficit de l'ingestion calorique sur la thermogenèse, sont réalisées.

L'enregistrement continu et simultané sur $48 \mathrm{~h}$ de la consommation d'oxygène, et de la prise alimentaire spontanée (fig. 2) illustre ces fluctuations et les déséquilibres, à court terme, qui en résultent. La consommation d'oxygène continue est oscillante. Elle l'est dans une marge étroite chez cet animal à la neutralité thermique et limité dans son activité. Chez un animal libre, l'exercice musculaire et, en ambiance froide, la thermogenèse de réchauffement pourront élever considérablement ces fluctuations. Parallèlement la prise alimentaire montre une double périodicité (Le Magnen et Devos, 1980). L'animal fait des repas et durant ces repas son bilan entrée-sortie est considérablement positif. La périodicité circadienne, bien connue, se superpose à cette périodicité des repas. L'animal mange de nuit trois à quatre fois ce qu'il mange de jour. Sur 6 rats et 38 enregistrements quotidiens, on constate l'excès de la prise sur la dépense durant les $12 \mathrm{~h}$ nocturnes, le déficit de jour (fig. 3) (Le Magnen et Devos, 1970 ; Le Magnen et Devos, 1984). De repas à repas de nuit, le bilan entre l'ingestion durant le repas et la dépense métabolique jusqu'au repas suivant, est positif. II est négatif de jour (fig. 4). En conséquence, l'animal augmente son poids (Kakolewski et al., 1971) et le contenu lipidique de sa carcasse de nuit (Panksepp, 1973). II perd, le jour sui- 


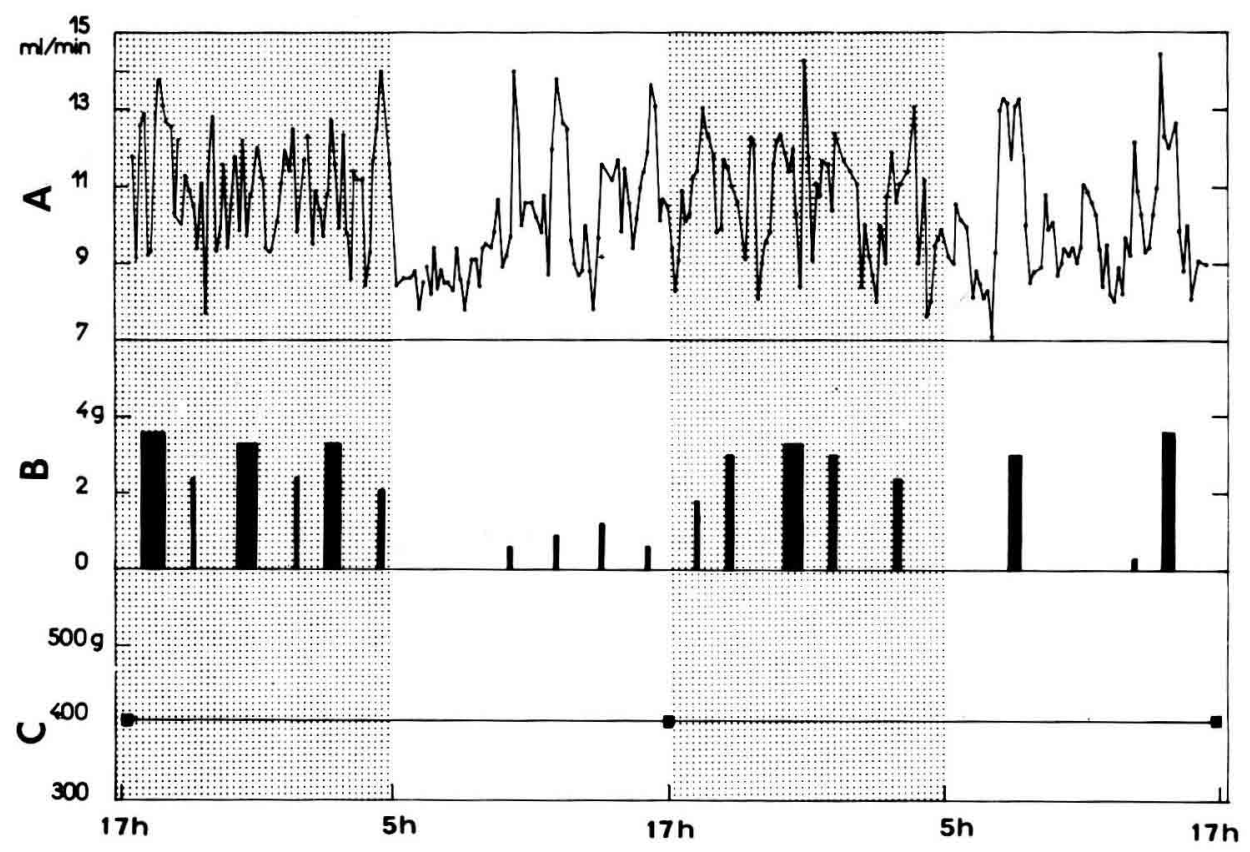

FIG. 2. - Enregistrement simultané durant $48 \mathrm{~h}$ chez un rat particulier; (Rat 10), de. haut en bas ; A) de la consommation d'oxygène; B) de la séquence alimentaire ; Cl du poids après $24 h$.

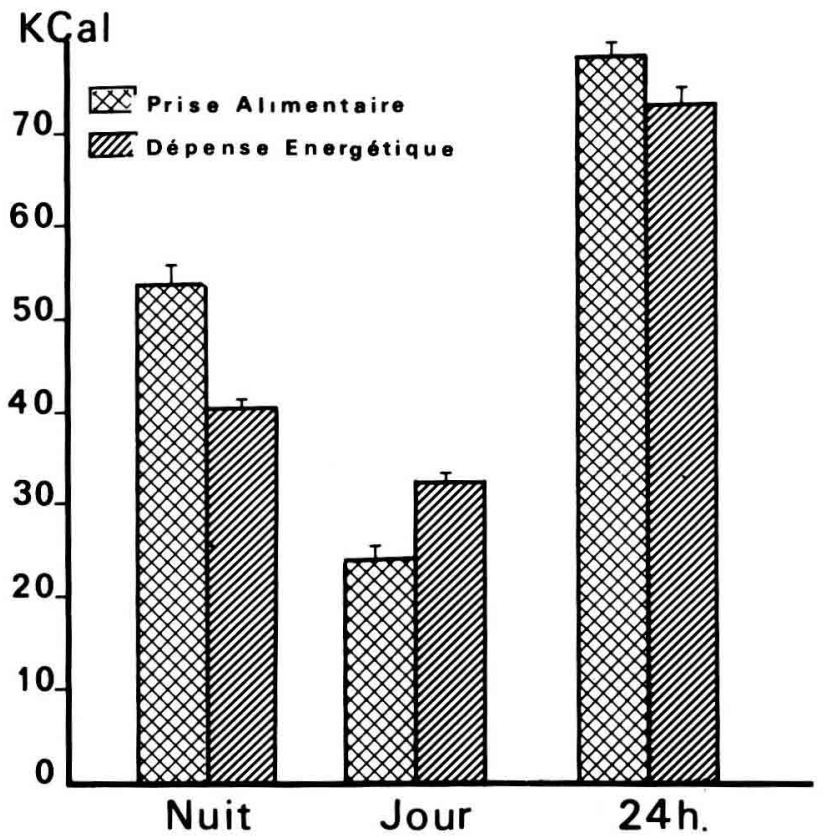

FIG. 3. - Moyennes de 38 jours chez six rats, des valeurs caloriques de la prise alimentaire et de la dépense métabolique concomitante en périodes nocturne et diurne et sur les $24 \mathrm{~h}$. Le bilan est fortement positif de nuit, négatif de jour. II est proche de l'équilibre sur les $24 \mathrm{~h}$. 


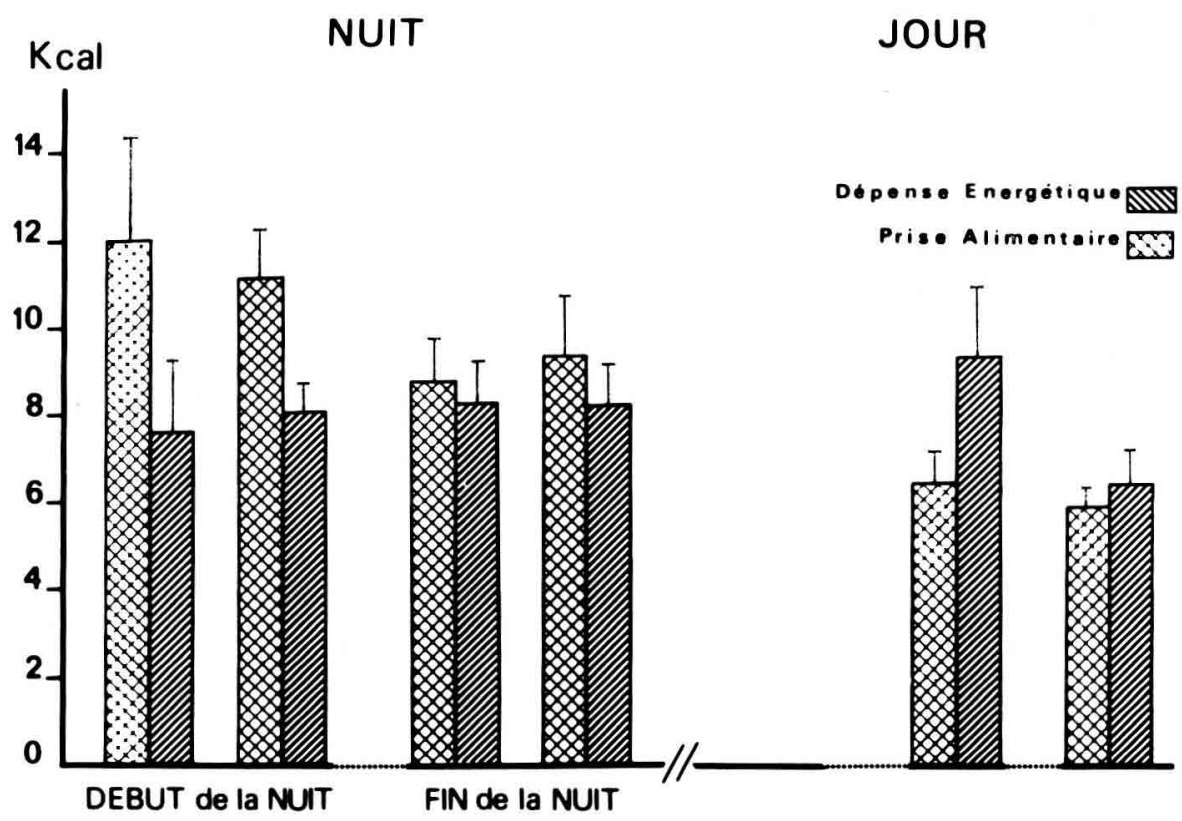

FIG. 4. - Bilans de nuit et de jour entre la prise calorique dans le repas et la dépense calorique du début de ce repas au déclenchement du suivant. Les bilans sont positifs de nuit, particulièrement au début de la nuit; ils sont négatifs de jour.

vant, ce poids et cette graisse accumulée la nuit. Cependant, le bilan mesuré sur les $24 \mathrm{~h}$ (fig. 3) est proche de l'équilibre et le poids mesuré de $24 \mathrm{~h}$ en $24 \mathrm{~h}$ (fig. 2) est approximativement constant. Les mêmes faits sont observés et peuvent se mesurer chez l'homme. Nous ingérons dans nos trois repas diurnes, deux fois notre dépense des $12 \mathrm{~h}$, mettons en réserve cette surcharge qui couvre la dépense énergétique nocturne.

\section{Les modèles de régulation.}

Ces déséquilibres instantanés et à court terme, conduisant à un équilibre du bilan et à une constance du poids à moyen et long termes, impliquent des mécanismes de régulation, de corrections et d'ajustement. Quatre sont concevables : 1) la thermogenèse s'ajuste à une prise alimentaire non contrôlée et " capricieuse " ;

2) les mécanismes déterminants de la prise alimentaire sont asservis par rétroaction à la thermogenèse ;

3) les mécanismes alimentaires, non commandés par la thermogenèse, ajustent néanmoins l'acquisition d'énergie à la dépense :

4) le compartiment des réserves, sa réplétion ou sa déplétion assurent la régulation par rétroaction sur la thermogenèse et/ou sur la prise alimentaire, ou les deux. 
a) L'ajustement de la thermogenèse à une prise alimentaire non contrôlée est a priori inconcevable et il est contraire à tous les faits. L'animal et même l'homme ne mangent pas n'importe quelle quantité, n'importe quand et n'importe quoi. Nous verrons que la prise alimentaire, en relation avec la dépense métabolique et avec le contenu calorique de l'aliment, est à la fois régulée et régulatrice.

b) La marge d'ajustement, en augmentation et réduction de la thermogenèse pour la réalisation de l'équilibre, est étroite. A jeun, elle n'est pas réductible et bien entendu le bilan négatif inévitable se traduit par la fonte des graisses. Sur l'autre versant, trois mécanismes de production d'extra-chaleur : les hormones thyroïdiennes, le tissu adipeux brun et l'exercice, sont susceptibles d'intervenir pour compenser un excès alimentaire sur la dépense courante; mais ces trois mécanismes d'élévation du métabolisme énergétique ont leurs fonctions propres qui excluent leur intervention comme régulateurs du bilan. En ambiance froide, par exemple la production d'extra-chaleur par le tissu adipeux brun ou thermogenèse sans frisson, permet le maintien de l'homéothermie. Seule l'augmentation de la prise alimentaire pourra alors rééquilibrer le bilan, et c'est en fait, expérimentalement, ce qui se passe. Il en est de même pour l'exercice musculaire. Rien ne démontre que l'on s'agite lorsqu'on a faim. C'est en fait exactement l'inverse. Chez le rat, la somnolence fait partie des symptômes dits "comportementaux " de la satiété et l'animal dort d'autant plus qu'il a plus mangé. A jeun, il est hyperactif.

Cependant, dans un cas particulier d'excès de l'ingestion sur la dépense métabolique, des données expérimentales sérieuses sembleraient avoir démontré l'intervention d'une production d'extra-chaleur par le tissu adipeux brun dans le sens d'une régulation du bilan (Rothwell et Stock, 1979). Le conditionnel est malheureusement prudent car une autre série de données, tend à démontrer le contraire (Barr et McCraken, 1982; Barr et al., 1983; Barr et McCraken, 1983 ; McCraken et Barr, 1983 ; Armitage et al., 1981a et b ; McCraken et Barr, 1982 ; Bestley et al., 1982 ; Hervey et Tobin, 1982).

Le cas particulier est le rat placé sur le régime dit de " cafétéria ». L'animal qui a libre accès à un choix d'aliments variés et hautement palatables augmente de 50 à $100 \%$ sa prise calorique quotidienne. Il gagne du poids et devient obèse (Rolls et Rowe, 1977 ; Sclafani et Gorman, 1977). La mesure de la consommation d'oxygène, comme l'établissement du bilan (prise calorique totale moins l'augmentation des lipides de la carcasse) permettent à Rothwell et Stock (Rothwell et Stock, 1982) d'affirmer la production d'une extra-chaleur, freinant l'établissement de l'obésité. Cette production d'une thermogenèse alimentaire régulatrice est due à une activation adrénergique du tissu adipeux brun. La mise en œuvre de l'activité thermogénique de ce tissu est directement appuyée par un ensemble de données : élévation de la température locale du tissu adipeux brun interscapulaire (Rothwell et Stock, 1979), hypertrophie du tissu, augmentation de sa réponse calorigénique à la noradrénaline, du contenu et du turnover de la noradrénaline (Rothwell et al., 1981) de la liaison de la guanosine diphosphate sur la membrane interne des mitochondries (Rothwell et al., 1982), l'élévation du débit circulatoire (Rothwell et Stock, 1981) et de la consommation d'oxygène du tissu. Cet ensemble de preuves convaincantes est mis en question par les données de Hervey et 
collaborateurs d'une part, (Hervey et Tobin, 1982) de McCraken et al. d'autre part. (McCraken et Barr, 1982 ; Bestley et al., 1982). Ces derniers auteurs, expérimentant sur la même souche de rat que Rothwell et collaborateurs, et effectuant exactement les mêmes mesures, concluent que l'élévation de la thermogenèse sur le régime de cafétéria ne dépasse pas le coût exothermique de la synthèse et du dépôt des graisses. D'autre part, sans démentir les autres données de Rothwell sur l'activation du tissu adipeux brun, ils montrent que le gain de poids de ce tissu est proportionnel à celui du tissu adipeux blanc (fig. 5).

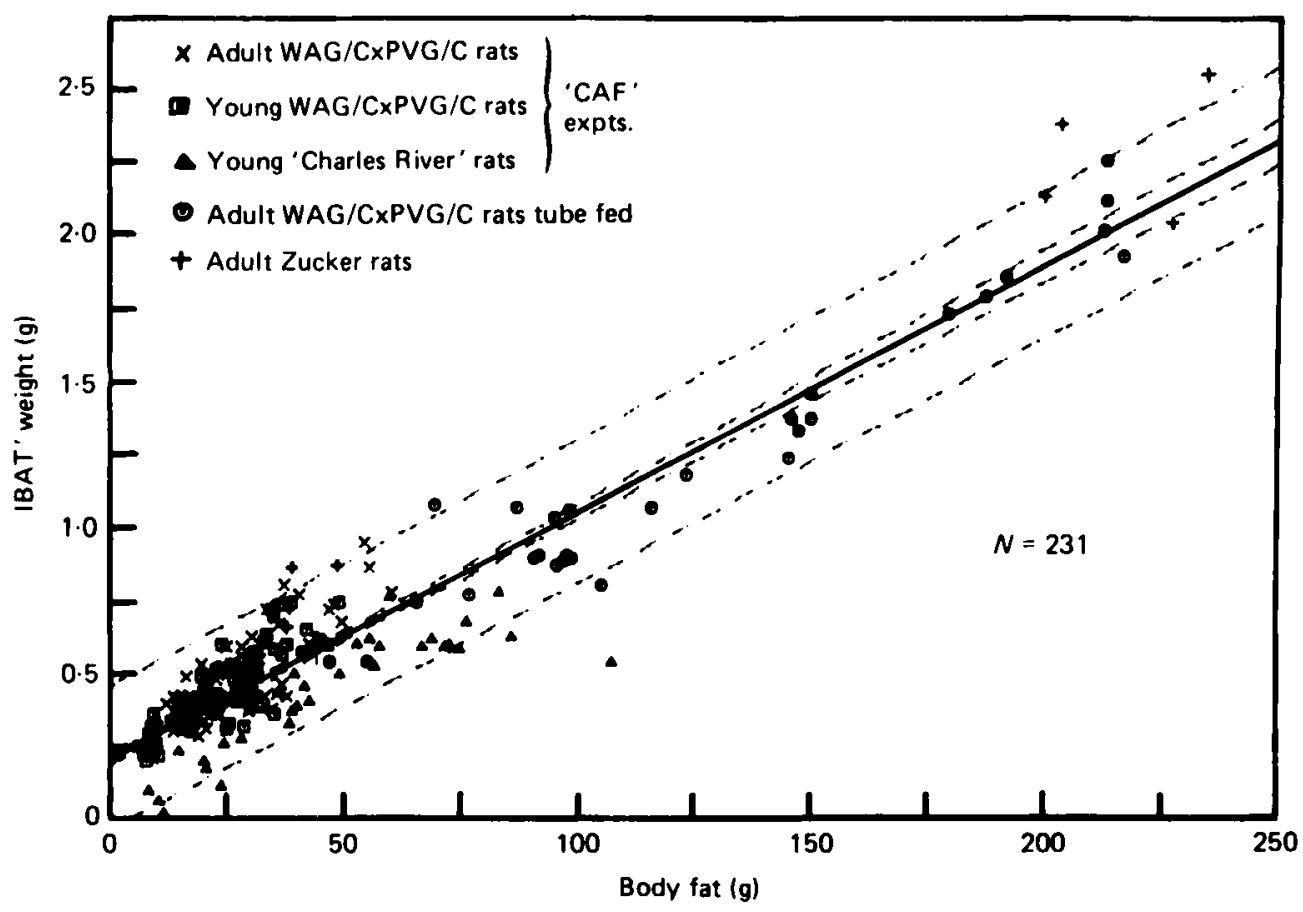

Regression of IBAT weight on total body fat for all experiments at $22^{\circ} \mathrm{C}$.

FIG. 5. - Régression linéaire entre la prise de poids du tissu adipeux brun et du tissu adipeux blanc chez le rat nourri par un régime de cafétéria (d'après Hervey et Tobin, J. Physiol. (London), 1982, $330: 71 \mathrm{P}-73 \mathrm{P})$.

La seconde notion suivant laquelle la prise alimentaire serait asservie et par conséquent commandée par le débit de perte d'énergie est sans fondement. Elle impliquerait que des détecteurs de ce débit, éventuellement représentés par la sensibilité thermique, dirigent la prise alimentaire. C'est là la vieille théorie thermostatique suivant laquelle on mangerait parce que l'on a froid ou pour éviter d'avoir froid, tandis que l'on cesserait de manger parce que l'on a trop chaud ou pour éviter d'avoir trop chaud. Cette théorie, comme certaines de ses résurgences 
récentes sous forme de théories dites " énergostatiques ", appartiennent à l'anecdote.

Tout démontre que l'effecteur principal sinon exclusif de la régulation du bilan énergétique est constitué par le mécanisme nerveux qui détermine le comportement alimentaire en réponse d'une part et à court terme, à la charge sanguine en glucose, d'autre part et à moyen ou long terme, aux mouvements de la masse adipeuse.

\section{Le mécanisme alimentaire.}

Deux systèmes de contrôle distincts déterminent à court terme la prise d'aliments et sa périodicité prandiale. Un mécanisme intervient pour déterminer le volume variable du repas depuis son initiation jusqu'au rassasiement. II fait intervenir essentiellement la réponse aux activités sensorielles orales de l'aliment et la rétroaction négative en réponse à l'aliment au niveau gastrique et/ou intestinal (Le Magnen, 1983). Ce contrôle périphérique permet à l'animal après un processus de conditionnement, d'anticiper dans son repas, de ses dépenses ultérieures. On peut considérer le repas comme la prise d'une réserve gastro-intestinale. Cette " charge prandiale » n'est pas dépendante de l'état nutritionnel, antérieur au déclenchement du repas. Dans les conditions de libre accès chez le rat, il n'existe pas de corrélation entre la dimension des repas et le temps qui s'est écoulé depuis le repas précédent (fig. 6) (Le Magnen et Devos, 1980). En revanche, il existe une

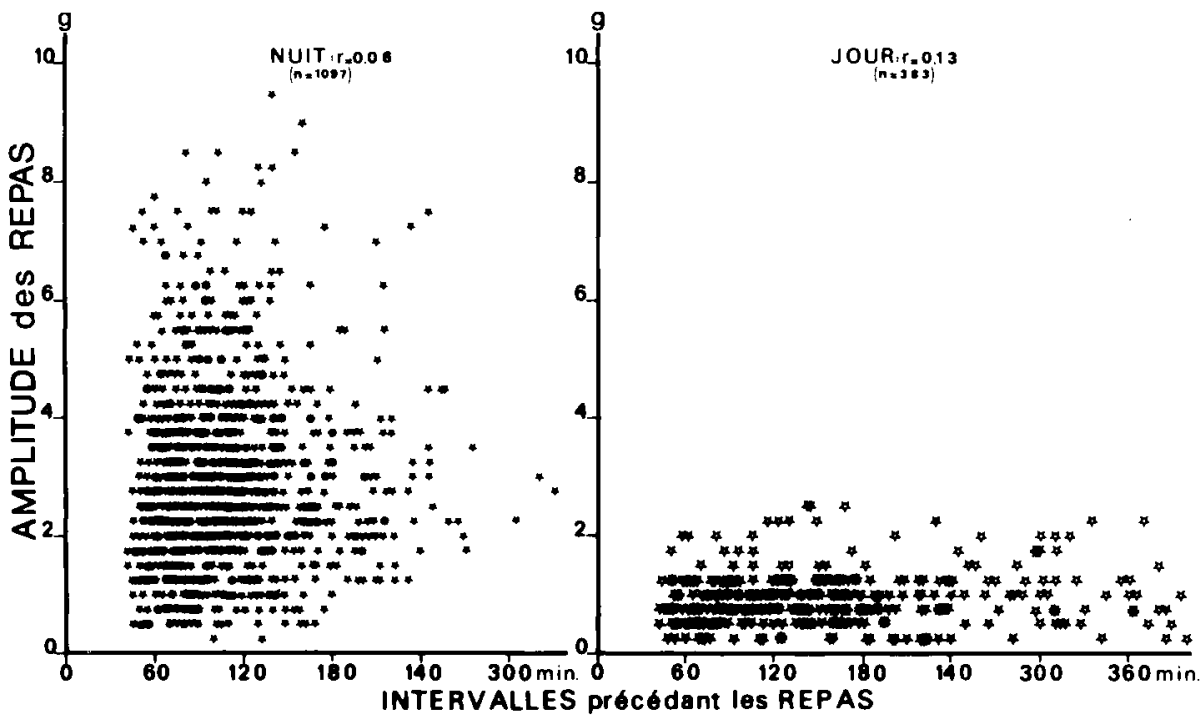

FIG. 6. - Absence de corrélation chez le rat ad libitum entre le volume variable des repas et la durée des intervalles qui précèdent le déclenchement de ces repas. Le mécanisme déterminant le volume des repas n'est donc pas affecté par la situation nutritionnelle au moment de son déclenchement. 
corrélation hautement significative dite « corrélation post-prandiale " entre l'ingestion calorique dans un repas et le temps qui s'écoule jusqu'au déclenchement du suivant (fig. 7). Ce fait révélait que le mécanisme initiateur du repas était dépendant d'une situation de déficit engendré par l'utilisation métabolique de la charge calorique d'un repas, depuis ce repas jusqu'au déclenchement du suivant.

Quel est ce déficit, ce signal ou stimulus à manger ? Nous avons apporté des preuves décisives que ce stimulus initiateur du " manger " chez le rat ad libitum, était constitué par la baisse de la charge sanguine en glucose (Louis-Sylvestre et Le Magnen, 1981).

La mesure continue de la glycémie et de la séquence alimentaire chez l'animal éveillé montre que le déclenchement de chaque repas est précédé d'une légère chute de la glycémie (fig. 8). Lorsque le repas est empêché par retrait de l'aliment, par exemple au début de la période nocturne, (fig. 9) cette chute de la glycémie s'accentue brutalement (Larue-Achagiotis et Le Magnen, 1983b). Le maintien d'une glycémie constante, autrement dit la régulation de la glycémie, requiert donc que l'animal mange. Au cours du cycle quotidien, l'animal mange à tout moment la quantité d'aliments juste nécessaire au maintien de sa glycémie. Une privation alimentaire de $3 \mathrm{~h}$ provoque une hypoglycémie de niveau variable suivant la position de cette privation de nuit et de jour (Larue-Achagiotis et Le Magnen, 1983a). Durant ces mêmes périodes de $3 \mathrm{~h}$, l'animal, disposant de son

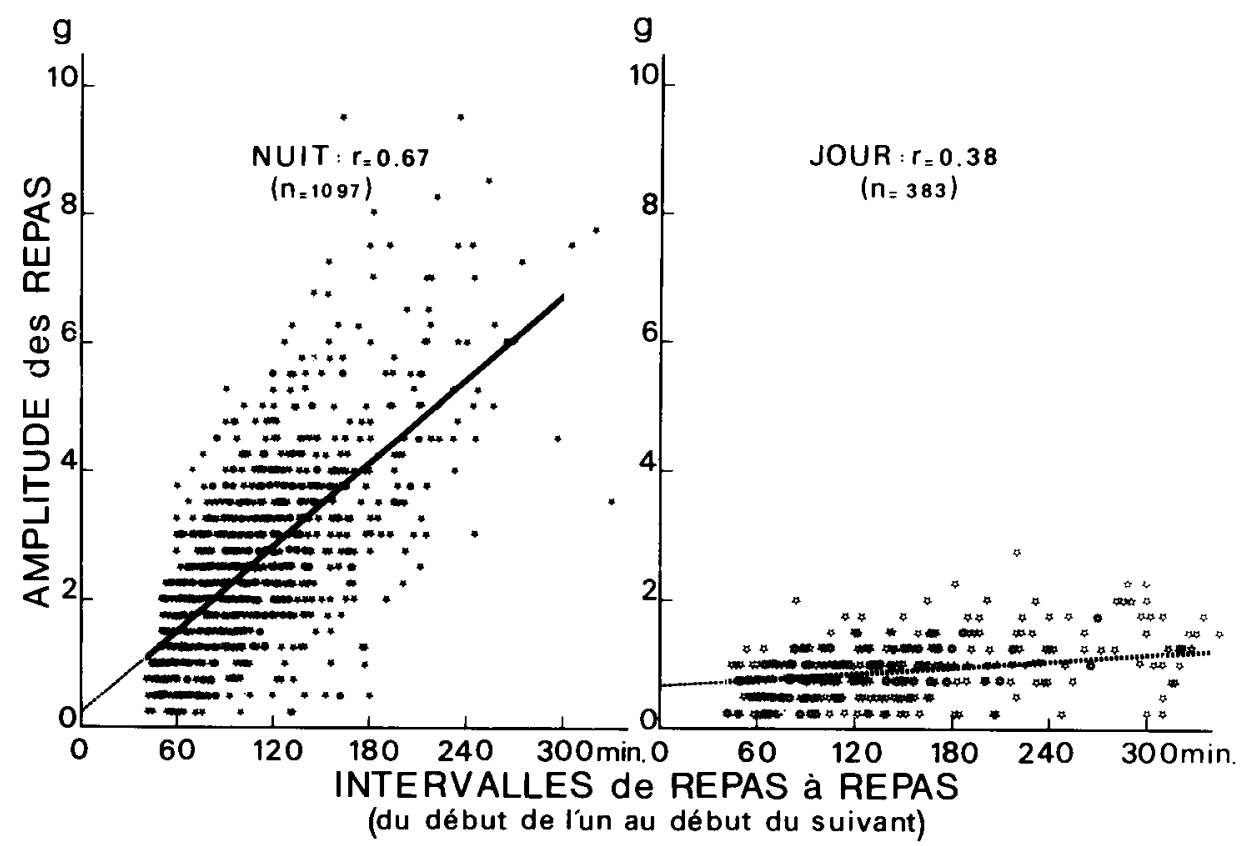

FIG. 7. - Corrélation positive et significative chez le rat ad libitum entre le volume des repas et la durée des intervalles qui suivent ces repas; différence de la relation entre périodes nocturne et diurne. Le mécanisme initiateur du repas, au contraire de celui qui détermine son volume, est donc dépendant de la situation nutritionnelle présente à la fin de l'intervalle post-prandial. 


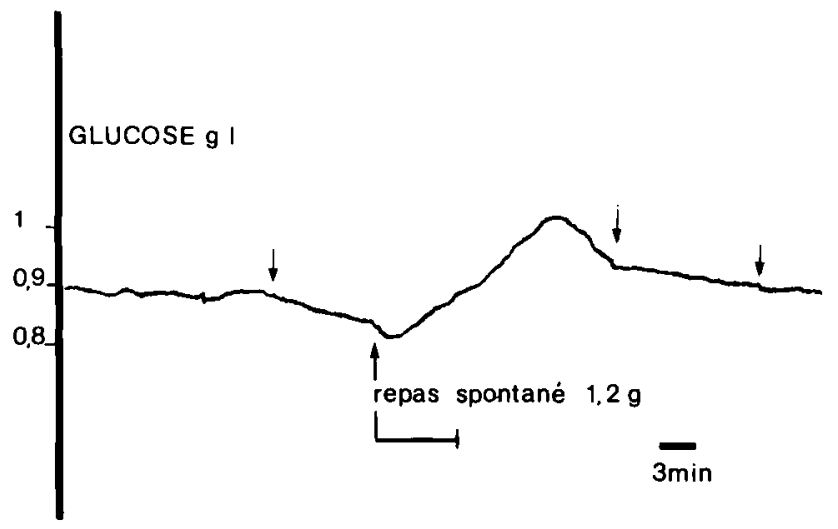

FIG. 8. - Enregistrement continu et simultané de la glycémie et de la séquence alimentaire. Le déclenchement spontané de chaque repas de nuit comme de jour est précédé d'une légère chute de la glycémie. Cette glycémie est constante depuis le retrait de I'hyperglycémie prandiale du précédent repas.

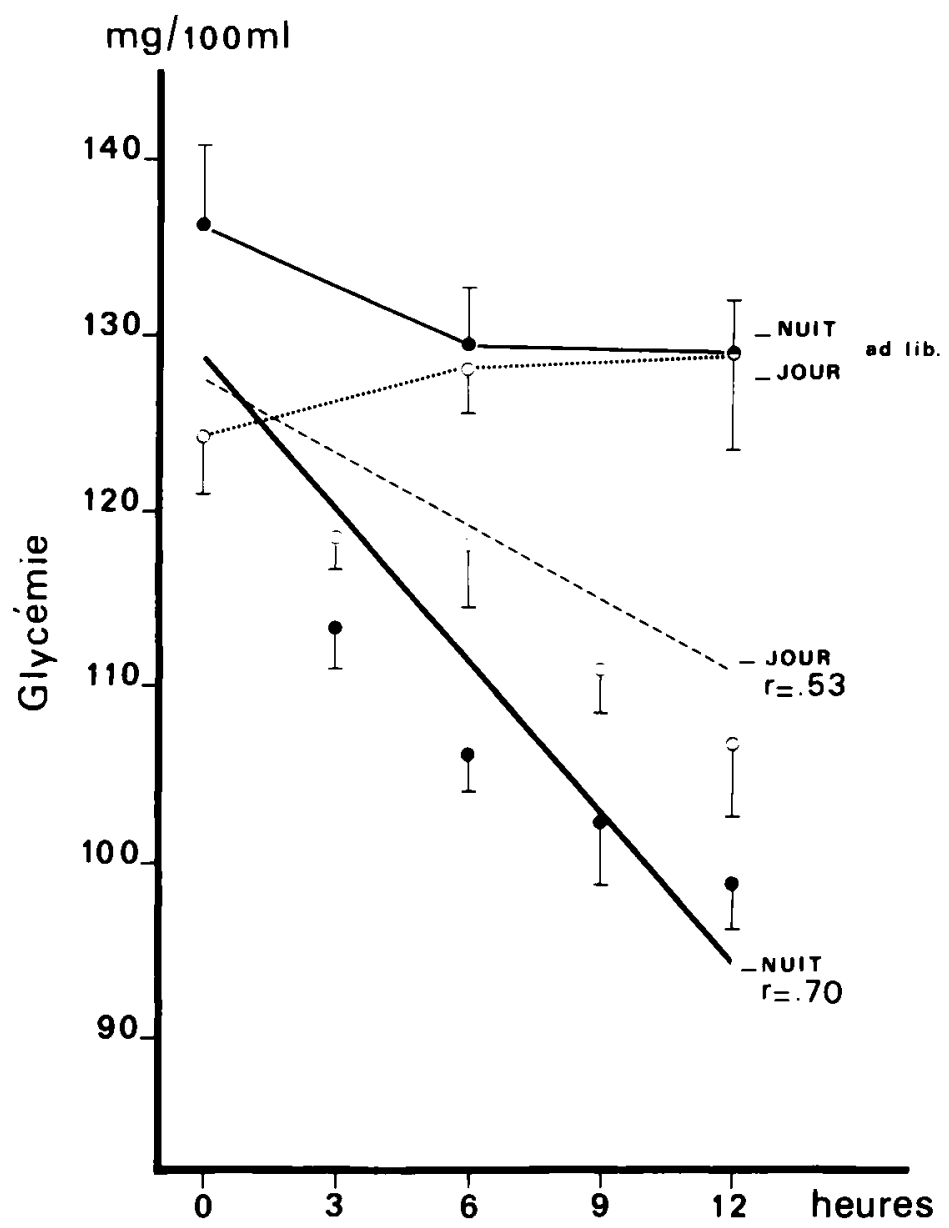

FIG. 9. - Chute de la glycémie chez le rat à jeun durant 12 h de nuit ou de jour. Cette hypoglycémie induite par la privation est beaucoup plus importante de nuit que de jour. Au début de la nuit, l'empêchement du premier repas entraîne une chute de la glycémie dès la première heure de jeûne. La prise d'aliment est donc une composante à moyen termè de la régulation de la glycémie. 

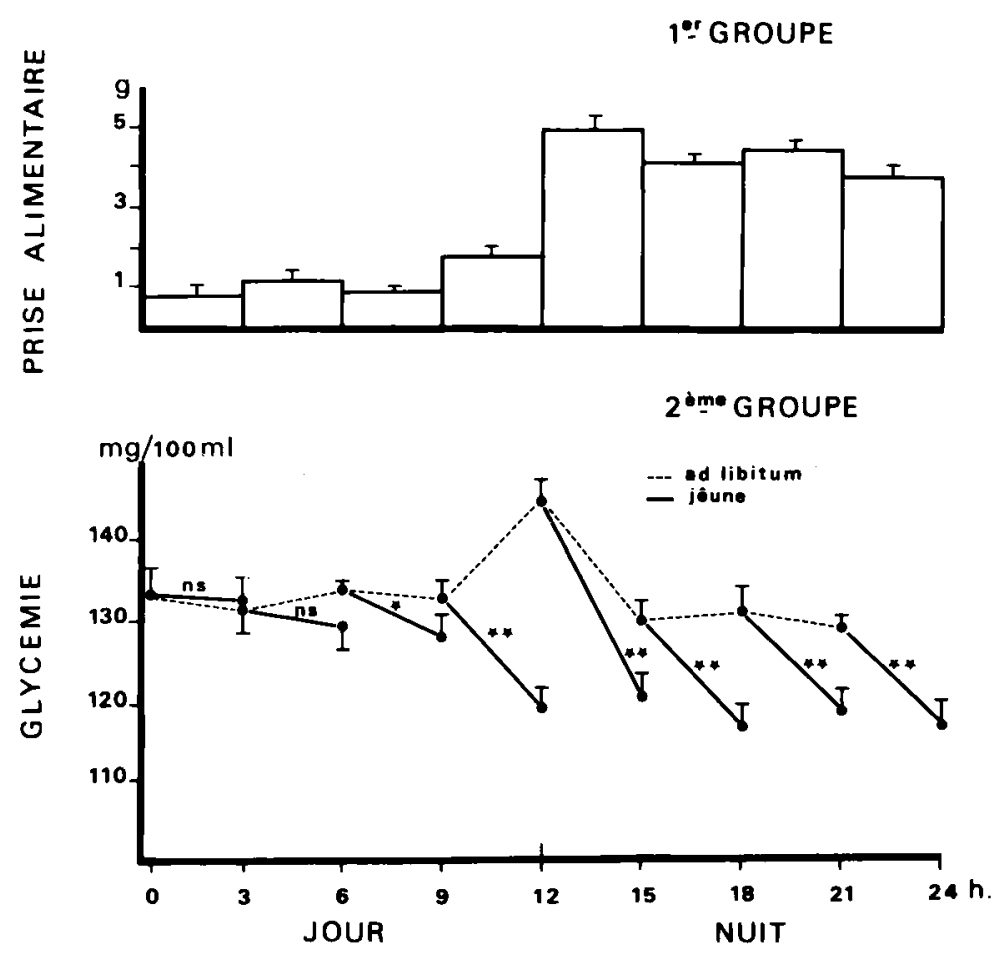

FIG. 10. - Chute de la glycémie provoquée par $3 \mathrm{~h}$ de privation alimentaire. Le niveau de I'hypoglycémie après $3 \mathrm{~h}$ est différent suivant la position de ce jeûne dans le nycthémère. La quantité d'aliments consommée en $3 \mathrm{~h}$ par des rats témoins est proportionnelle à l'hypoglycémie qu'entraîne la privation durant les $3 \mathrm{~h}$ à ce moment du nycthémère. Le rat mange donc à tout moment la quantité d'aliments juste nécessaire au maintien de sa glycémie.

aliment, en mange une quantité proportionnelle à cette chute de glycémie lorsque la nourriture lui est retirée (fig. 10).

La prise alimentaire est donc une composante de la régulation de la glycémie. Un grand nombre de données suggèrent que le déficit de disponibilité cellulaire de glucose associé à cette hypoglycémie est actif sur des sites centraux, sites plus spécifiquement sensibles que le reste du cerveau à l'approvisionnement en glucose. Ils sont dits pour cette raison (à tort ou à raison) " glucorécepteurs ". De nombreux arguments, on le sait, ont été fournis pour la localisation de ces glucorécepteurs dans la région latérale de l'hypothalamus où se projettent d'autre part, les afférences sensorielles olfactives et gustatives (Le Magnen, 1983).

Ce mécanisme rend compte pour une part de l'équilibre du bilan des entrées et sorties de glucose du distributeur de ce métabolite aux tissus qu'est le sang. Cependant, il ne rend pas compte à lui seul de la régulation du bilan d'énergie se traduisant à moyen ou long terme par l'équilibre du bilan des gains et pertes de la réserve adipeuse. Un second mécanisme y intervient : nous avons démontré qu'un mécanisme liporégulateur module le mécanisme d'initiation alimentaire en fonction des états de réplétion ou de déplétion de la masse adipeuse. 


\section{Le mécanisme liporégulateur.}

L'existence d'un système liporégulateur est depuis longtemps clairement démontrée. La nature de ce mécanisme et son rôle dans la régulation du bilan énergétique n'ont été élucidés que très récemment.

L'existence d'un mécanisme corrigeant des élévations ou réductions de la masse adipeuse au-delà d'une certaine marge et, par conséquent, assurant la constance moyenne de cette masse est démontrée par l'observation d'une réversibilité des obésités et maigreurs expérimentalement induites (May et Beaton, 1968 ; MacKay et al., 1940 ; Panksepp et al., 1975). L'administration quotidienne d'insuline retard ou la perfusion continue d'insuline normale (fig. 11) (LarueAchagiotis, en préparation), la stimulation électrique répétée (Steinbaum et Miller, 1965 ; Stephens et Morrissey, 1975) de l'hypothalamus latéral, enfin, on l'a vu, un régime de cafétéria, induisent une hyperphagie et une obésité. A la cessation du traitement ou d'un gavage (Cohn et Joseph, 1962) qui a aussi établi l'obésité, l'animal est aphagique ou hypophagique. II est en lipolyse constante et par conséquent perd du poids. Cette hypophagie et la perte du poids cessent lorsque l'animal est revenu à son poids initial. La perte de poids induite par le jeûne ou une restriction prolongée, est suivie, à la réalimentation, d'une hyperphagie et d'une lipogenèse continue (Pénicaud et Le Magnen, 1980a). Elles disparaissent lorsque l'animal a réparé sa maigreur.
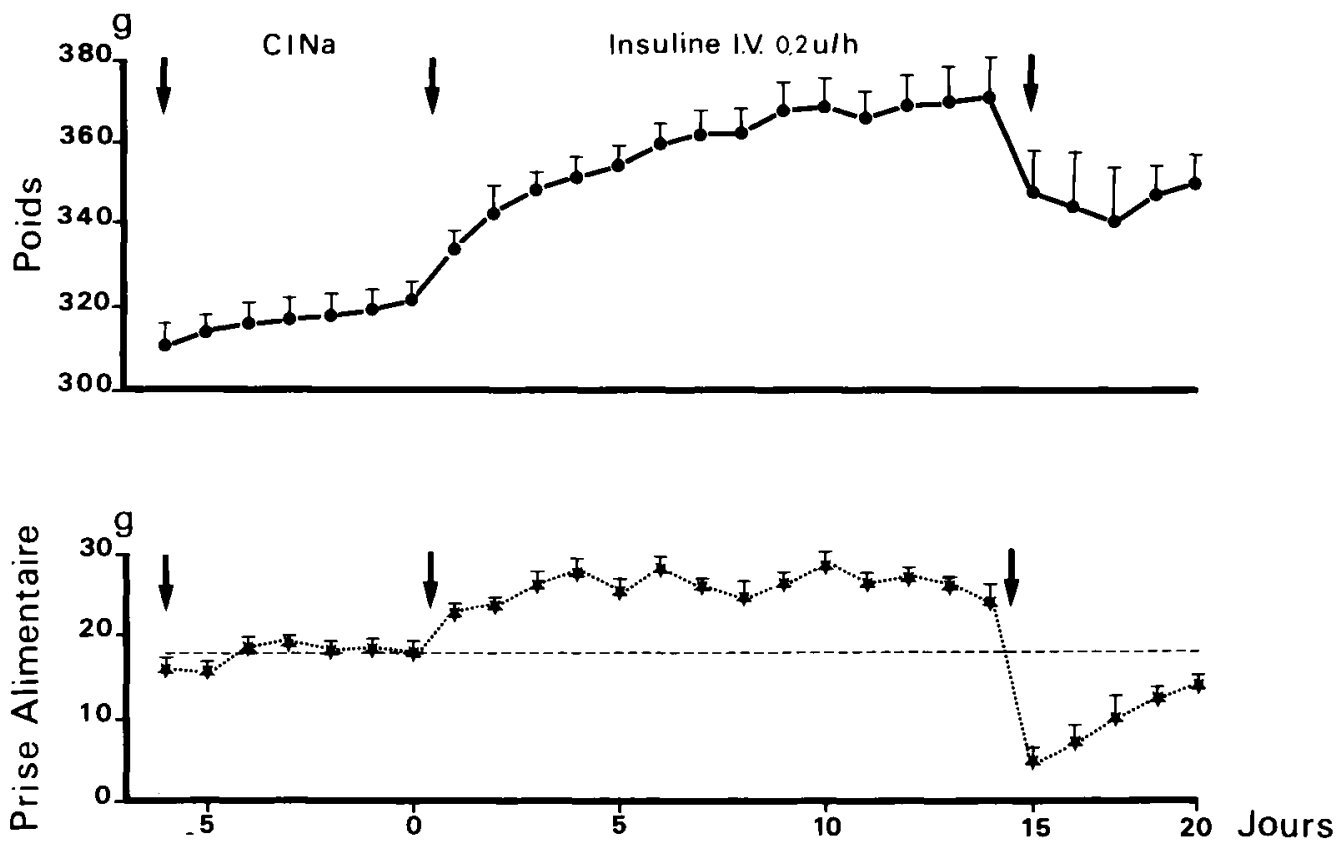

FIG. 11. - Hyperphagie et obésité induites chez le rat par une perfusion continue intraveineuse d'insuline. A la cessation du traitement, l'animal est hypophagique et perd du poids jusqu'au retour à son poids initial. 
La nature exacte de ce mécanisme liporégulateur a été étudiée dans le cycle lipogenèse-lipolyse quotidien du rat où il est physiologiquement en œuvre.

Nous avons mentionné plus haut qu'un bilan positif et un gain de poids nocturnes, un bilan négatif et une perte de poids diurnes étaient observés chez le rat (fig. 3). Sur les $24 \mathrm{~h}$ le bilan proche de l'équilibre et le poids approximativement constant de jour en jour témoignent d'une compensation régulatrice entre les situations nocturne et diurne. Une corrélation négative existe entre excès du bilan nocturne et déficit du bilan diurne (fig. 12) (Le Magnen et Devos, 1982). L'examen des bilans de repas à repas durant les deux périodes démontre que de nuit la fréquence d'initiation des repas est accélérée par une double utilisation métabolique de l'aliment ingéré dans chaque repas, représentée par la couverture du métabolisme énergétique à laquelle s'ajoute le prélèvement pour la lipogenèse (Le Magnen et Devos, 1984). De jour, cette fréquence est ralentie par un double apport de fuel aux tissus, représenté par les aliments ingérés auxquels s'ajoute l'oxydation des lipides mobilisés, celle-ci épargnant l'utilisation de glucose (Balasse, 1971 ; Balasse et Neef, 1974).

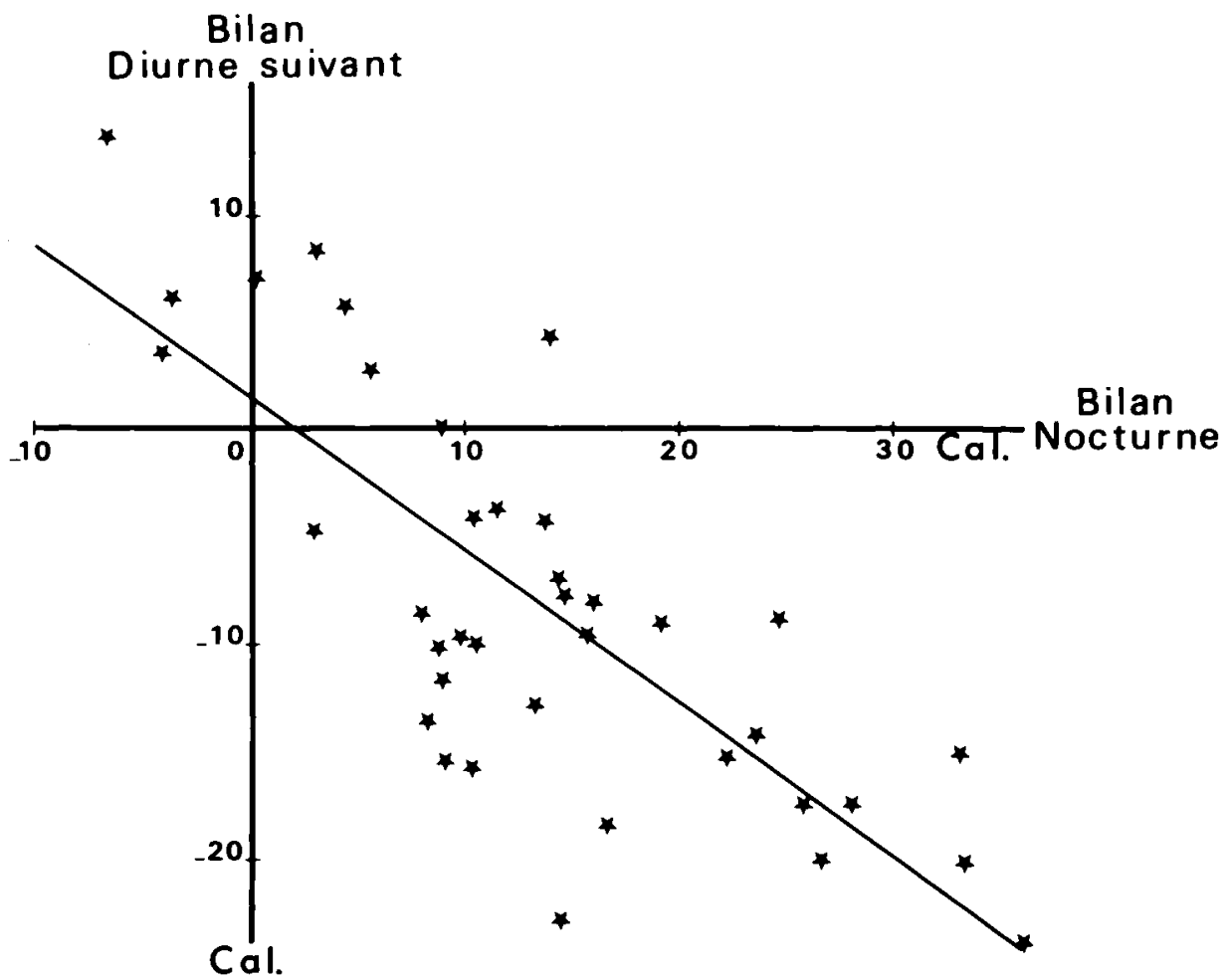

FIG. 12. - Corrélation négative entre l'excès nocturne de la prise alimentaire sur la dépense métabolique et le déficit de ce bilan, le jour suivant. Cette compensation entre les situations métaboliques des deux phases du cycle est en jeu dans l'établissement de l'équilibre du bilan des $24 \mathrm{~h}$.

Reproduction, Nutrition, Développement $n^{\circ} 5$ B $1984 .-2$. 
Un mécanisme neuroendocrinien primaire détermine cette séquence métabolique quotidienne et son effet sur le mécanisme alimentaire. Le rat de nuit, I'homme de jour (Aparicio et al., 1974 ; Sensi et al., 1973 ; Gibson et al., 1975) sont hyperinsuliniques. Les insulines basale et stimulée sont élevées (fig. 13) ainsi que la tolérance au glucose (fig. 14) et la sensibilité tissulaire à l'insuline (fig. 15) (Le Magnen et al., 1973 ; Louis-Sylvestre, 1978 ; Hara et Saito, 1980 ; Pénicaud et Le Magnen, 1980b ; Sakata et al., 1977 et 1982). Ce complexe est déterminant de la lipogenèse. De jour chez le rat, de nuit chez l'homme, la lipolyse est associée à un hypoinsulinisme, à une intolérance relative au glucose.

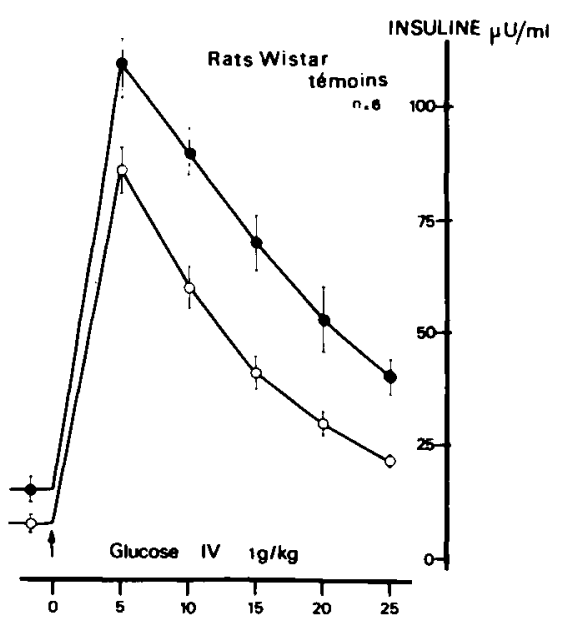

FIG. 13. - L'insulinémie basale et stimulée par une charge de glucose est plus élevée de nuit que de jour.

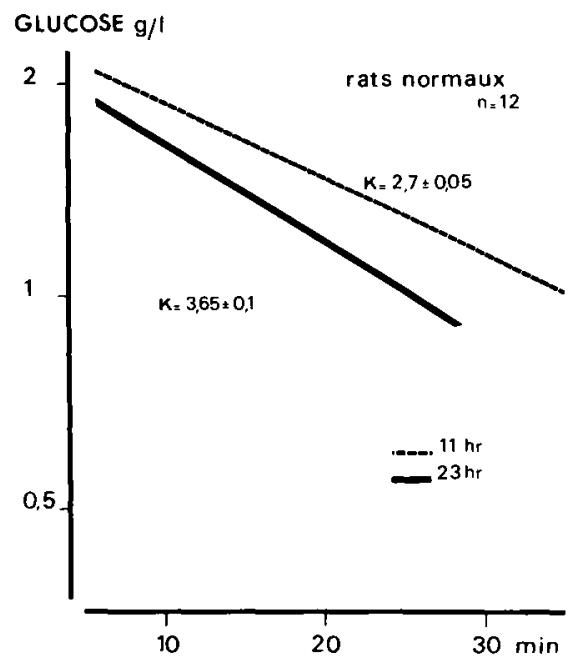

FIG. 14. - Le rat manifeste une tolérance au glucose élevée (coefficient K) en période nocturne, une intolérance relative au glucose en période diurne.

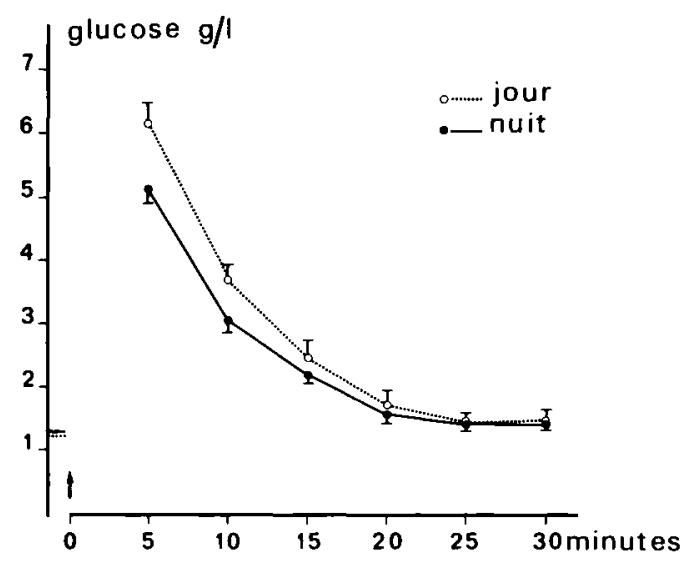

FIG. 15. - L'hypoglycémie provoquée par une administration d'insuline est plus profonde de nuit que de jour, suggérant une sensibilité tissulaire à l'insuline plus élevée en période nocturne. 
Un mécanisme lipostatique et ses relations avec le mécanisme alimentaire conduisant à l'équilibre du bilan d'énergie sont donc clairement identifiés. Une lipogenèse, induite par un hyperinsulinisme endogène ou, expérimentalement, par de l'insuline exogène, accélère le fonctionnement du mécanisme de stimulation alimentaire par l'élévation de la captation tissulaire de glucose. Elle provoque donc I'hyperphagie. Cette lipogenèse fonctionnelle au-delà d'un certain seuil et/ou expérimentalement à la cessation du traitement induit une lipolyse correctrice. Cette lipolyse et l'oxydation des métabolites lipidiques, par l'épargne de glucose, ralentissent l'intervention d'une stimulation alimentaire. Elles provoquent donc I'hypophagie. En dessous de la marge régulée de la masse adipeuse, une lipolyse imposée par le jeûne ou la restriction induit la lipogenèse correctrice manifestée à la réalimentation. Cette lipogenèse correctrice entraîne également I'hyperphagie.

II est bien établi que la mise en œuvre de la lipolyse comme de la lipogenèse est contrôlée par des voies nerveuses descendantes sympathiques et parasympathiques antagonistes. La lipolyse est dépendante des efférences adrénergiques directes au tissu adipeux, des efférences sympathiques aux médullo-surrénales responsables des décharges de catécholamines, enfin des efférences sympathiques au pancréas, inhibitrices, on le sait, de décharges d'insuline. La lipogenèse est dépendante d'une levée de ces activations et inhibitions sympathiques et de la facilitation vagale des décharges d'insuline du pancréas. L'enregistrement des décharges dans le nerf surrénalien et dans la branche pancréatique du vague a clairement démontré cette activation sympathique et la réduction d'activité parasympathique dans les situations lipolytiques (sous l'effet du 2-désoxy-D-glucose par exemple), et l'inverse dans les situations de lipogenèse (Niijima, 1975). In vitro, les décharges d'insuline en réponse au glucose sont le résultat d'un équilibre entre noradrénaline et acétyl-choline ajoutées au milieu (Campfield et Smith, 1983).

\section{Rôle des noyaux médio-ventraux de l'hypothalamus dans le méca- nisme liporégulateur.}

Cette commande nerveuse et donc le mécanisme lipostatique sont gouvernés par les noyaux médio-ventraux de l'hypothalamus. La destruction de ces noyaux abolit l'activation sympathique de la lipolyse qui, par conséquent, en dépend. La stimulation électrique de ces noyaux chez l'animal éveillé effectivement stimule I'élévation des acides gras libres circulants et la perte de poids (Shimazu, 1981). Chez l'animal lésé, l'induction de la lipolyse par le 2-désoxy-D-glucose, l'exercice ou le jeûne, est altérée (Nishizawa et Bray, 1978 ; Bray et al., 1981). Le turnover tissulaire de la noradrénaline est réduit (Van der Tuig et al., 1982 ; Seydoux et al., 1979 et 1981 ; Girardier et al., 1976) et d'autres preuves sont apportées (Inoue et Bray, 1980) d'une réduction du tonus sympathique. Cette altération de la stimulation adrénergique de la lipolyse démasque et fait prévaloir l'activité parasympathique, en particulier sur le pancréas endocrine. L'animal lésé est donc hyperinsulinique et hypoglucagonémique (Chikamori et al., 1980). Cette situation se traduit en période nocturne chez le rat par l'exagération de la lipogenèse et de l'hyperphagie 
fonctionnelle de cette période. De jour, le complexe lipolytique hypoinsulinique et hypophagique a disparu. L'animal hyperinsulinique et hyperphagique sur les $24 \mathrm{~h}$, parce qu'il a perdu le mécanisme de compensation nuit-jour, prend rapidement du poids et devient obèse. La cyclicité alimentaire (Pénicaud et Le Magnen, 1984) et neuro-endocrinienne a disparu chez ces animaux en phase dynamique de prise de poids (fig. 16). La séquence alimentaire, identique de nuit et de jour, reproduit
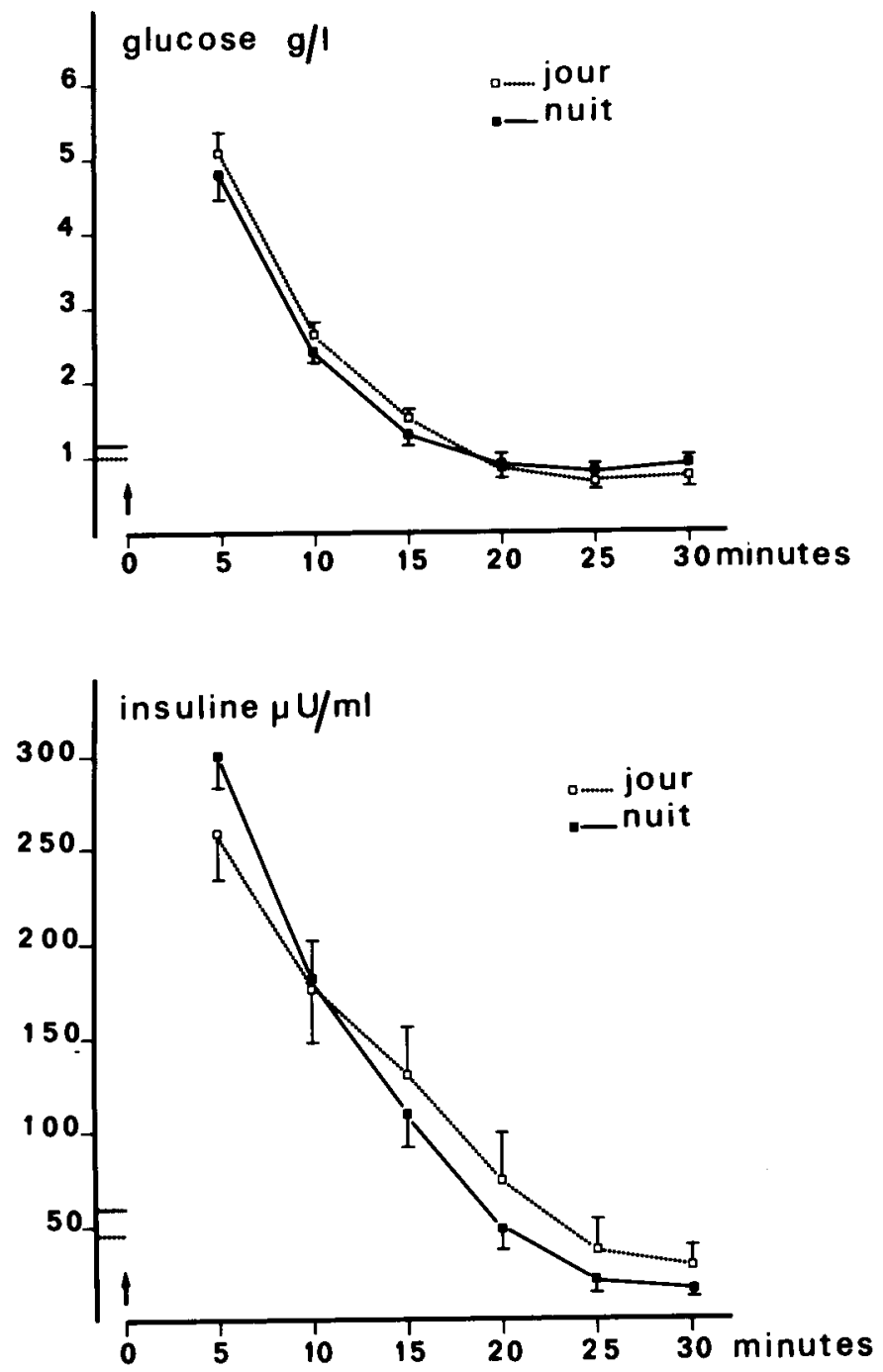

FIG. 16. - Chez l'animal hyperphagique et devenant obèse après la lésion hypothalamique, l'hyperinsulinisme et la tolérance au glucose de la période nocturne sont augmentés et s'étendent à la période diurne. La lésion a donc fait disparaître le complexe métabolique et alimentaire diurne, ce qui entraîne l'hyperphagie des 24 h et l'obésité. 
celle d'animaux intacts traités par une perfusion continue d'insuline (LarueAchagiotis et Le Magnen, 1983b (fig. 17). Mais, contrairement à ces animaux intacts dont l'obésité induite par l'insuline exogène est réversible à la cessation du traitement, les lésés ont perdu la capacité de limiter la réplétion de leur compartiment adipeux et de corriger sa sur-réplétion. Mis à jeun et amaigris au-dessous de leur poids pré-opératoire, ils reviennent, à la réalimentation, à leur poids d'obèses. Les animaux génétiquement obèses : rats Zucker et souris ob/ob, ne sont pas sensiblement différents des rats hypothalamiques. Ils sont également hyperinsuliniques et hyperphagiques sur les $24 \mathrm{~h}$ (Bray et York, 1979). Chez eux également des preuves ont été apportées d'une faiblesse du tonus sympathique, en particulier, d'une réduction du turnover de la noradrénaline dans le tissu adipeux (Young et Langsberg, 1983 ; Levin et al., 1983 ; Rohner-Jeanrenaud et al., 1983).

Une question majeure reste à poser. Comment les noyaux médio-ventraux de I'hypothalamus ou des structures connectées à ces noyaux, sont-ils informés des

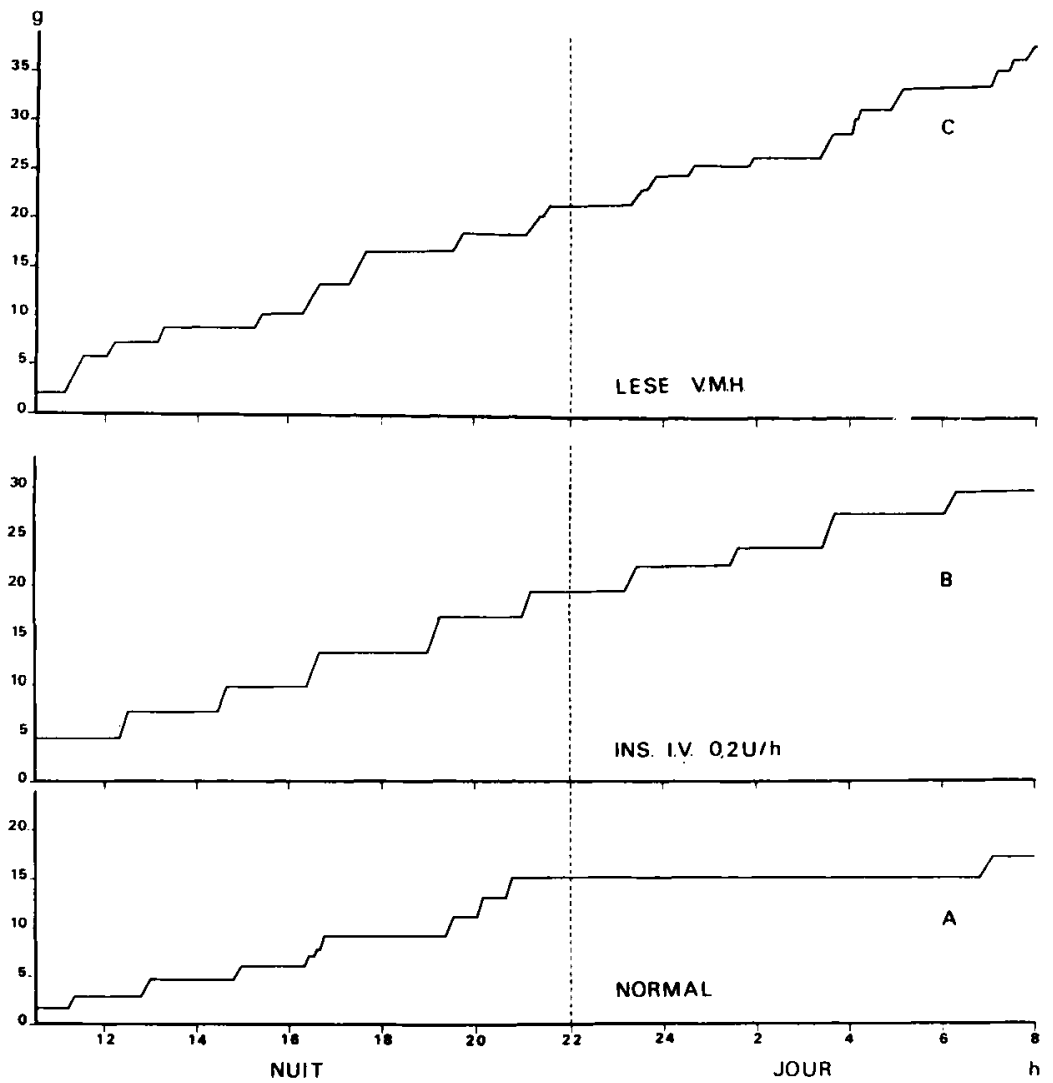

FIG. 17. - Séquences alimentaires comparées chez le rat normal, chez le rat hypothalamique et le rat traité en continu par l'insuline. L'hyperinsulinisme continu par l'insuline exogène reproduit la séquence alimentaire du rat hypothalamique. 
mouvements de la masse adipeuse périphérique et, en réponse à ces mouvements, mettent-ils en œuvre les efférences nerveuses correctrices ? Des voies nerveuses afférentes issues de tous les dépôts adipeux et fournissant cette information sont inconcevables et sont inconnues. On devait donc faire I'hypothèse qu'un facteur associé aux mouvements de lipogenèse-lipolyse et variant avec lui pouvait être le véhicule de l'information et de la réponse centrales. Un ensemble d'arguments a été réuni qui suggère que ce facteur est la concentration plasmatique de l'insuline.

L'hyperinsulinisme endogène comme exogène chronique a, manifestement, une double action périphérique et centrale, action en sens opposé sur le tissu adipeux. L'activité périphérique est la lipogenèse, dépendante de l'insuline. L'activité centrale chronique est le développement de la stimulation centrale et sympathique de la lipolyse, correctrice de l'activité lipogénique de cette même insuline. Lorsque l'insuline est administrée en continu par la voie intra-ventriculaire, chez le singe et chez le rat, et que son activité périphérique est ainsi exclue, la lipolyse et l'hypophagie sont induites sans délai et l'animal maigrit en dessous de son poids initial (Woods et al., 1979 ; Davis et Brief, 1981). Lorsque l'insuline est perfusée à la périphérie, l'activité lipogénique prévaut ; mais son activité centrale sous-jacente est révélée par l'atténuation progressive de l'efficacité lipogénique de l'hormone au cours du traitement et à mesure que la masse adipeuse s'élève (LarueAchagiotis et Le Magnen, 1983b). Elle est surtout démasquée à la cessation du traitement par la lipolyse, l'hypoinsulinisme, l'hypophagie et la chute de poids jusqu'au retour au poids initial. L'hypoinsulinisme chronique dû au jeûne, et peutêtre aussi au diabète, pourrait-être une image en miroir de cette double et inverse action centrale et périphérique de l'insuline, l'activité centrale étant ici responsable de la lipogenèse et de I'hyperphagie correctrices.

Quelle est cette action centrale de l'insuline au niveau vraisemblable du VMH ? Un modèle a été proposé par nous, dont la vérification expérimentale est en cours dans divers laboratoires. Des résultats préliminaires le confirment. II est fondé sur l'identification au sein du système nerveux central de récepteurs à l'insuline et de leur ségrégation au sein de diverses structures en particulier de la région médiale de l'hypothalamus (Havrankova et al., 1981 ; Van Houten et Posner, 1981). Depuis longtemps, des glucorécepteurs, distincts de ceux présents dans la région latérale, ont été localisés dans cette même région médio-ventrale, en particulier par la lésion par l'aurothioglucose chez la souris (Oomura, 1976 ; Debons et al., 1970a, b, 1974).

On sait que les récepteurs à l'insuline des adipocytes, hépatocytes et autres tissus cibles de l'insuline manifestent une sous- et sur-régulation (" down and up regulation "). Une élévation de l'insuline circulante réduit le nombre et l'affinité des récepteurs et par conséquent la sensibilité du tissu à l'insuline. L'hypoinsulinémie a l'action inverse. L'obèse hyperinsulinique développe la sous-régulation et une résistance à l'insuline (Olefsky et Kolterman, 1981) ; le jeûneur et le diabétique insulino-dépendants, la sur-régulation. Chez l'homme, on a démontré un cycle quotidien des récepteurs à l'insuline périphérique : une augmentation de la liaison durant la phase lipolytique nocturne, une réduction durant la phase lipogénique, hyperinsulinique diurne (Beck-Nielsen et Pedersen, 1978). Rien ne permet 
de penser que les insulinorécepteurs cérébraux, contrairement aux périphériques, ne sont pas sous- et sur-régulés. On doit donc penser (et des résultats préliminaires semblent le vérifier) que les insulinorécepteurs médio-ventraux répondent à l'hyperinsulinisme du sang ou du liquide cérébrospinal par une réduction de leur affinité pour l'insuline, et à un hypoinsulinisme par le mouvement inverse. Ces changements de la liaison de l'insuline sur les membranes des neurones glucosensibles seraient à l'origine d'une sensibilisation ou désensibilisation de ces neurones à la glucopénie. Ces variations sont parallèles et proportionnelles à l'élévation ou à la réduction de la masse adipeuse sous l'effet périphérique des hyper- et hypo-insulinismes. Elles fourniraient le signal des réponses correctrices médiées par les voies nerveuses descendantes, sympathiques et parasympathiques.

Journées Ingestion, Digestion, Absorption de l'Association française de Nutrition, Strasbourg, 20-21 octobre 1983.

\section{Références}

APARICIO N., PUCHULU F. E., GAgliARDINO J. J., RUIZ M. D., LLORENS J. M., RUIZ J., LAMAS A., DE MIGUEL R., 1974. Circadian variation of the blood glucose, plasma insulin and human growth hormone levels in response to an oral glucose load in normal subjects. Diabetes, 23, 132-137.

ARMitAGE G., HERVEY G. R., ROLLS B. J., ROWE E. A., TOBIN G., 1981a. Energy balance in " cafeteria »-fed adult rats. J. Physiol. (London), 316, 48P.

ARMITAGE G., HERVEY G. R., ROLLS B. J., ROWE E. A., TOBIN G., 1981b. Energy balance in young « cafeteria »-fed rats. J. Physiol. (London), 317, 48P.

BALASSE E. O., 1971. Effect of free fatty acids and ketone bodies on glucose uptake and oxidation in the dog. Horm. Metab. Res., 3, 403-409.

BALASSE O. E., NEEF M. A., 1974. Operation of the " glucose-fatty acid cycle " during experimental elevations of plasma free fatty acid levels in man. Eur. J. clin. Invest., 4, 247-252.

BARR H. G., McCRAKEN K. J., 1982. Absence of " diet-induced thermogenesis " in growing rats kept at $29^{\circ}$ and offered a varied diet. Proc. Nutr. Soc., 41, 63A.

BARR H. G., McCRAKEN K. J., 1983. No " diet-induced thermogenesis " in young lean Zucker rats offered a varied diet. Proc. Nutr. Soc., 42, $102 \mathrm{~A}$.

BARR H. G., GRAY R., McCRAKEN K. J., 1983. Computed intakes of essential nutrients by growing rat offered a varied diet. Proc. Nutr. Soc., 42, 2A.

BESTLEY J. W., BRAMLEY P. N., DOBSON P. M. S., MAHANTY A., TOBIN G., 1982. Energy balance in " cafeteria "-fed young "Charles River " Sprague-Dawley rats. J. Physiol. (London), 330, 70P.

BECK-NIELSEN H., PEDERSEN O., 1978. Diurnal variation in insulin binding to human monocytes. J. clin. Endocrinol. Metab., 47, 385-390.

BRAY G. A., INOUE S., NISHIZAWA Y., 1981. Hypothalamic obesity. The autonomic hypothesis and the lateral hypothalamus. Diabetologia, 20 (suppl.), 366-378.

BRAY G. A., YORK D. A., 1979. Hypothalamic and genetic obesity in experimental animals : an autonomic and endocrine hypothesis. Physiol. Rev., 59, 719-809.

CAMPFIELD L. A., SMITH F. J., 1983. Neural control of insulin secretion : interaction of norepinephrine and acetylcholine. Am. J. Physiol., 244, R629-634.

CHIKAMORI K., NISHIMURA F., SUEHIRO F., SATO K., MORI H., SAITO S., 1980. Alterations in glucagon secretion in obese rats with hypothalamic lesions. Horm. Metab. Res., 12, 56-59.

COHN D., JOSEPH D., 1962. Influence of body weight and body fat on appetite of " normal " lean and obese rats. Yale J. Biol. Med., 34, 598-607. 
DAVIS J. D., BRIEF D. J., 1981. Chronic IV insulin infusion reduces food intakes and body weight in rats. Abst. Soc. Neurosci, 7, 655.

DEBONS A. F., KRIMSKY I., FROM A., CLOUTIER R. J., 1970a. Site of action of gold thioglucose in the hypothalamic satiety center. Am. J. Physiol., 219, 1397-1402.

DEBONS A. F., KRIMSKY I., FROM A., CLOUTIER R. J., 1970b. Gold thioglucose induction of obesity: significance of focal gold deposits in hypothalamus. Am. J. Physiol., 219, $1403-1408$.

DEBONS A. F., KRIMSKY I., FROM A., PATTINIAN H., 1974. Phlorizin inhibition of hypothalamic necrosis induced by gold thioglucose. Am. J. Physiol., 226. 574-578.

GIBSON T., STIMMER L., JARRETT J., RUTLAND P., SHIU M., 1975. Diurnal variation in the effect of insulin on blood glucose plasma non-esterified fatty acids and growth hormone. Diabetologia, 11, 83-88.

GIRARDIER L., SEYDOUX J., CAMPFIELD L. A., 1976. Control of A and B cells in vivo by sympathetic nervous input and selective hyper-or hypoglycemia in dog pancreas. $J$. Physiol. (London), 256, 801-814.

HARA E., SAITO M., 1980. Diurnal changes in plasma glucose and insulin responses to oral glucose load in rats. Am. J. Physiol., 238, E463-467.

HAVRANKOVA J., BROWNSTEIN M., ROTH J., 1981. Insulin and insulin receptors in rodent brain. Diabetologia, 20 (suppl.), 268-273.

HERVEY G. R., TOBIN G., 1982. Brown adipose tissue weight in relation to rat body fat content. J. Physiol. (London), 330, 71P-73.

INOUE S., BRAY G. A., 1980. Role of the autonomic nervous system in the development of ventromedial hypothalamic obesity. Brain Res. Bull., 5 (Suppl. 4), 119-126.

KAKOLEWSKI J. W., DEAUX E., CHRISTENSEN J., CASE B., 1971. Diurnal patterns in water and food intake and body weight changes in rats with hypothalamic lesions. Am. J. Physiol., 221, 711-718.

LARUE-ACHAGIOTIS C., LE MAGNEN J., 1983a. In rats, the effects of $3 \mathrm{~h}$ deprivation on blood glucose level and on subsequent intake are correlated throughout the day. ICPFFI, Melbourne.

LARUE-ACHAGIOTIS C., LE MAGNEN J., 1983b. Effets de l'administration chronique d'insuline sur la prise alimentaire et le gain de poids chez le rat. X/1" réunion A.F.N., Strasbourg.

LE MAGNEN J., 1983. Body energy balance and food intake: a neuroendocrine regulatory mechanism. Physiol. Rev., 63, 314-386.

LE MAGNEN J., DEVOS M., 1970. Metabolic correlates of the meal onset in the free food intake of rats. Physiol. Behav., 5, 805-811.

LE MAGNEN J., DEVOS M., 1980. Parameters of the meal pattern in rats : their assessment and physiological significance. Neurosci. Biobehav. Res., 4 (Suppl. 1), 1-11.

LE MAGNEN J., DEVOS M., 1982. Daily body energy balance. Physiol. Behav., 29, 807-811.

LE MAGNEN J., DEVOS M., 1984. Meal to meal energy balance. Physiol. Behav., 32 (in press).

LE MAGNEN J., DEVOS M., GAUDILLIERE J. P., LOUIS-SYLVESTRE J., TALLON S., 1973. Role of a lipostatic mechanism in regulation by feeding of energy balance in rats. J. comp. physiol. Psychol., 84, 1-24.

LEVIN B. E., TRISCARI J., SULLIVAN A. C., 1983. Altered sympathetic activity during development of diet-induced obesity in rat. Am. J. Physiol., 244, R 347-355.

LOUIS-SYLVESTRE J., 1978. Feeding and metabolic patterns in rats with truncular vagotomy or transplanted beta cells. Am. J. Physiol., 235, E 119.

LOUIS-SYLVESTRE J., LE MAGNEN J., 1981. A fall in blood glucose level precedes meal onset in free-feeding rats. Neurosci. Biobehav. Rev., 4 (suppl. 1), 13-16.

McCRACKEN K. J., BARR H. G. 1982. Energy balance and body fat changes in young " cafeteria "fed rats kept at $24^{\circ} \mathrm{C}$. J. Physiol. (London), 330, 69-70P.

MCCRACKEN K. J., BARR H. G., 1983 . No impairment in efficiency of energy utilization in young growing rats kept at $24^{\circ}$ and offered a varied diet. Proc. Nutr. Soc., 42, $3 \mathrm{~A}$.

MacKAY E. M., CALLAWAY J. W., BARNES R., 1940. Hyperalimentation in normal animals produced by protamine insulin. J. Nutr., 20, 59-66.

MAY K. K., BEATON J. R., 1968. Hyperphagia in the insulin-treated rat. Proc. Soc. exp. Biol. Med., 127, 1201-1204. 
NIIJIMA A., 1975. The effect of 2-deoxy-D-glucose and D-glucose on the efferent discharge rate of sympathetic nerves. J. Physiol. (London), 251, 231-243.

NISHIZAWA Y., BRAY G. A., 1978. Ventromedial hypothalamic lesions and mobilization of fatty acids. J. clin. Invest., 61, 714-721.

OLEFSKY J. M., KOLTERMAN O. G., 1981. The insulin receptor in physiological and pathological conditions, 463-488. In ANDREANI D., De PIRRO R., LAURO R., OLEFSKY J. M., ROTH J. Current views on insulin receptors. Acad. Press.

OOMURA Y., 1976. Significance of glucose, insulin and free fatty acids on the hypothalamic feeding and satiety neurons, 145-158. In NOVIN D., WYRWICKA W., BRAY G. A. Hunger : basic mechanisms and clinical implications. Raven Press (NY).

PANKSEPP J., 1973. Reanalysis of feeding patterns in the rat. J. comp. physiol. Psychol., 82, 78-94.

PANKSEPP J., POLLACK A., KROST K. P., MEEKER R., RITTER M., 1975. Feeding in response to repeated protamine zinc insulin injections. Physiol. Behav., 14, 487-493.

PÉNICAUD L., LARUE-ACHAGIOTIS C., LE MAGNEN J., 1983. Endocrine basis for weight gain after fasting or VMH lesion in rats. Am. J. Physiol., 245, E 246-252.

PÉNICAUD L., LE MAGNEN J. 1980a. Recovery of body weight following starvation or food restriction in rats. Neurosci. Biobehav. Rev., 4 (suppl. 1), 47-52.

PENICAUD L., LE MAGNEN J., 1980b. Aspects of the neuroendocrine bases of the diurnal metabolic cycle in the rats. Neurosci. Biobehav. Rev., 4 (suppl. 1), 39-42.

ROHNER-JEANRENAUD F., HOCHSTRASSER A. C., JEANRENAUD B., 1983. Hyperinsulinemia of preobese and obese fa/fa rats is partly vagus nerve-mediated. Am. J. Physiol., 244 (Endocrinol. Metab. 7), E317-E322.

ROLLS B. J., ROWE E. A., 1977. Dietary obesity; permanent changes in body weight (abstr.). J. Physiol. (London), 272, 2P.

ROTHWELL N., STOCK M., 1979. A role for brown adipose tissue in diet-induced thermogenesis. Nature, 281, 31-34.

ROTHWELL N., STOCK M. J., 1981. Influence of noradrenaline on blood flow to brown adipose tissue in rats exhibiting diet-induced thermogenesis. Pflügers Archiv., 389, 237-242.

ROTHWELL N. J., STOCK M. J., 1982. Energy expenditure derived from measurements of oxygen consumption and energy balance in hyperphagic cafeteria-fed rats. J. Physiol. (London), 324, 59P.

ROTHWELL N. J., STOCK M. J., TYZBIR R. S., 1982. Energy balance and mitochondrial function in liver and brown fat of rats fed " cafeteria " diets of varying protein content. J. Nutr., 112, 1663-1672.

ROTHWELL N. J., STOCK M. J., WYLLIE M. G., 1981. Na+-K+-ATPase activity and noradrenaline turnover in brown adipose tissue of rats exhibiting diet-induced thermogenesis. Biochem. Pharmacol., 30, 1709.

SAKATA T., FUKUSHIMA M., KODAMA J., FUCHIMOTO H., 1977. Light-dark patterns in running wheels activity in rats during chronic administration of theophylline. Physiol. Behav., 19, 397.401.

SAKATA T., FUKUSHIMA M., TSUTSUI K., ARASE K., FUJIMOTO K., 1982. Theophylline disrupts diurnal rhythms of humoral factors with loss of meal cyclicity. Physiol. Behav., 28, 641648.

SCLAFANI A., GORMAN A. N., 1977. Effect of age, sex and prior body weight on the development of dietary obesity in adult rats. Physiol. Behav., 18, 1021-1026.

SENSI S., CAPANI F., CARADONNA P., DELL'ACQUA G. B., 1973. Circadian rhythm of immunoreactive insulin under glycemic stimulus. J. interdiscipl. Cycle Res., 4, 1-14.

SEYDOUX J., BRUNSMANN M. J. A., JEANRENAUD B., GIRARDIER L., 1979. Alphasympathetic control of glucose output of mouse liver perfused in situ. Am. J. Physiol., 236, E323-327.

SEYDOUX J., ROHNER-JEANRENAUD F., ASSIMACOPOULOS-JEANNET F., JEANRENAUD L., GIRARDIER L., 1981. Functional disconnection of brown adipose tissue in hypothalamic obesity in rats. Pflügers Archiv., 390, 1-4.

SHIMAZU T., 1981. Central nervous system regulation of liver and adipose tissue metabolism. Diabetologia, 20 (Suppl.), 343-356. 
STEINBAUM E. A., MILLER N. E., 1965. Obesity from eating elicited by daily stimulation of hypothalamus. Am. J. Physiol, 208, 1-5.

STEPHENS D. N., MORRISSEY S. M., 1975. Hypothalamic stimulation induces acid secretion, hypoglycemia and hyperinsulinemia. Am. J. Physiol., 228, 1206-1209.

VAN DER TUIG J. G., KNEHANS A. W., ROMSOS D. R., 1982. Reduced sympathetic nervous system activity in rats with ventromedial hypothalamic lesions. Life Sci., 30, 913-920.

VAN HOUTEN M., POSNER B. I., 1981. Cellular basis of direct insulin action in the central nervous system. Diabetologia, 20 (Suppl.), 255-267.

WOODS S. C., LOTTER E. C., McKAY L. D., PORTE D. Jr., 1979. Chronic intracerebroventricular infusion of insulin reduces food intake and body weight of baboons. Nature, London, 282, 503-505.

YOUNG G., LANGSBERG L., 1983. Diminished sympathetic nervous system activity in genetically obese ob/ob mice. Am. J. Physiol., 245, 148-154. 